\title{
USING THE PHYSICAL OPTICS APPROXIMATION FOR ESTIMATING THE LIGHT SCATTERING PROPERTIES OF LARGE DUST PARTICLES FOR LIDAR APPLICATIONS
}

\author{
Alexander Konoshonkin ${ }^{* 1}$, Natalia Kustova ${ }^{1}$, Anatoli Borovoi ${ }^{1}$, Alexandra Tsekeri ${ }^{2}$, Josef \\ Gasteiger $^{3}$ \\ ${ }^{I}$ V.E. Zuev Institute of Atmospheric Optics SB RAS, Tomsk 634055, Russia \\ ${ }^{2}$ Institute for Astronomy Astrophysics Space Applications and Remote Sensing, Athens 15236, Greece \\ ${ }^{3}$ Faculty of Physics, University of Vienna, Vienna 1010, Austria \\ *Email:sasha_tvo@iao.ru
}

\begin{abstract}
The physical optics approximation was used to solve the light scattering problem of large dust particles with irregular shape. The comparison between the physical optics approximation and the DDA method was made for particles with volume-equivalent size parameters of 44.21. A full light scattering matrix (Mueller matrix) was calculated for particles with volume-equivalent size parameters from 44 up to 220 for two wavelengths: 0.532 and $1.064 \mu \mathrm{m}$. The dependences of the lidar and depolarization ratio, and extinction coefficient on the number of spatial orientations of the particle were obtained. It was shown that the elements of the light backscattering matrix obey power laws.
\end{abstract}

\section{INTRODUCTION}

Since the interpretation of the lidar signal is the inverse light scattering problem, it requires the calculation of the light scattering properties of the particles. In the case of large dust particles with realistic irregular shapes, the calculation of the scattering of light becomes a non-trivial task. This is due to the fact that the requirement for computational resources of numerical exact methods (FDTD, DDA) increases significantly with increasing the particle size. In this report, dust scattering properties are obtained within the framework of the physical optics approximation (PO), which has worked well for the solution of the light scattering of large atmospheric ice particles. The computational complexity of the PO does not increase with increasing the particle size, on the contrary, the larger the particle, the more accurate the approximation.

\section{NUMERICAL RESULTS}

Since the physical optics approximation provides satisfactory results for particles with size much larger than the wavelength, it suffices to show its applicability for particles with smaller sizes and thus define the lower limit of its applicability. Further, for any larger size accuracy will not be worse. In this report, the particle with the volumeequivalent size parameters $x=44.21$ was chosen as the minimum size. There

$$
x=2 \pi r_{v} / \lambda, r_{v}=\sqrt[3]{3 V / 4 \pi},
$$

where $r_{v}$ is the volume equivalent radius of the particle and $V$ is volume of the particle. The PO calculations are performed for two lidar wavelengths of 0.532 and 1.064 , and the refractive index was chosen as $m=1.48+i 0.0$ and $m=$ $1.6+i 0.0$, respectively. The particle shape was taken from [1], shape $F$.

Comparison of the solutions using the ADDA [2] and the PO [3] is presented in Figs. 1 and 2. It can be seen that the solutions agree well with each other. However, in the exact backward scattering direction, the solution essentially depends on the number of orientations.

Due to the requirements for computing resources of the ADDA code, it was possible to obtain a solution only for 288 orientations. The physical optics approximation is much faster, so we analyzed the dependence of lidar characteristics on the number of particle orientations using the PO, see Fig. 3. Figure 3 shows that the lidar and depolarization ratios essentially depend on the number of orientations, and in this case there is no convergence even at 4608 orientations, despite this fact, 72 orientations are sufficient to correctly calculate the extinction coefficient. Nevertheless, based on the estimation of [4], we believe that 4608 orientations are quite enough. Here we mean special averaging over only two Euler angles $\beta$ and $\gamma$, averaging over the third Euler angle $\alpha$ is performed analytically. 
It should be noted that the PO is an iterative method. At the first iteration, the solution is found only for external specular reflection, then it is refined at each iteration, see [5] for details. The convergence of the method on the number of iterations is presented in Fig. 4.
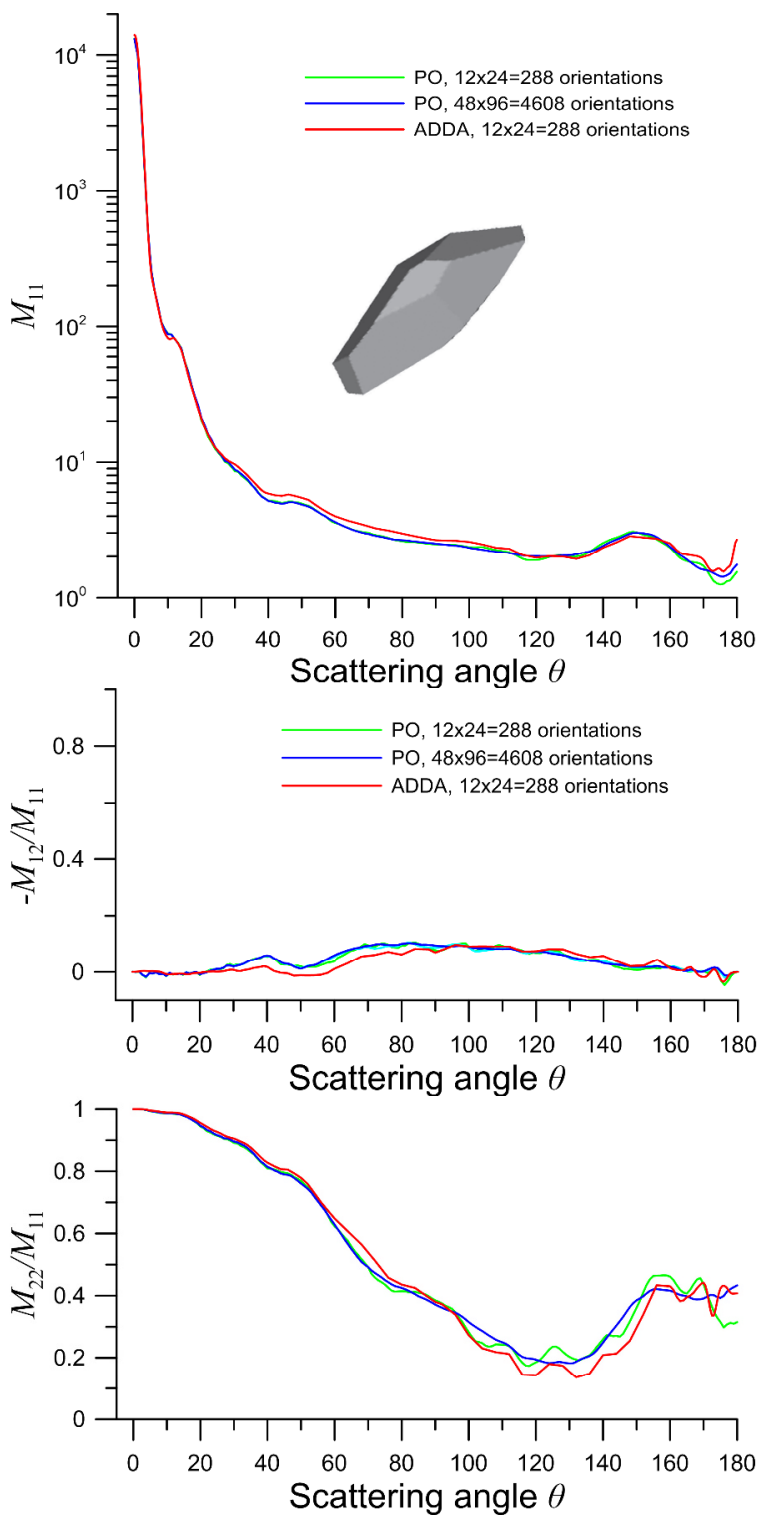

Figure 1. Elements of the Mueller matrix for a randomly oriented dust particle calculated within the physical-optics approximation and discrete dipole approximation for $0.532 \mu \mathrm{m}$.

For the calculations, we chose 7 iterations of the PO and 4608 special orientations. Figure 5 shows the dependence of the $M_{11}$ element of the light scattering matrix in the vicinity of the backward scattering direction on the particle size. We see that the interference oscillations are not significant and can be neglected (see Fig. 6), which allow us to increase the step of the particle size incrementation. Solution for particles with sizes from 40 up to 220 is presented in Fig. 7.
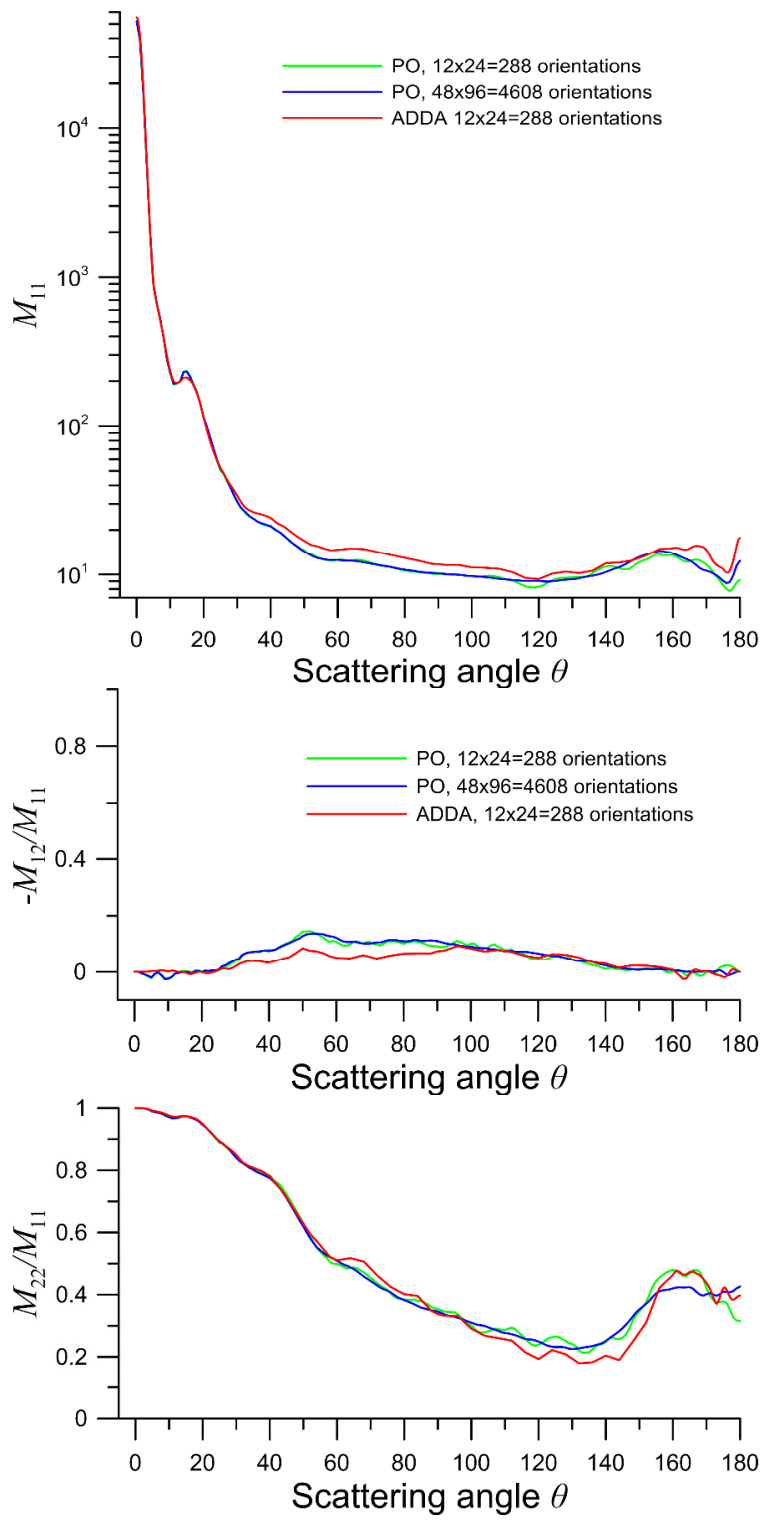

Figure 2. The same as Fig. 1, but for $1.064 \mu \mathrm{m}$.

Generally speaking, with a doubling in size it is necessary to increase the number of orientations four times. Therefore, the calculation for size $x=200$ requires 115200 orientations.

However, Fig. 8 clearly shows that the solution in the exact backscattering direction, that is important for the lidar application, can be well 
approximated by power functions similar to ice particles, see [6] for details. Note that this is shown only if we neglect the absorption effect. When the absorption is taking into account the approximation can change.
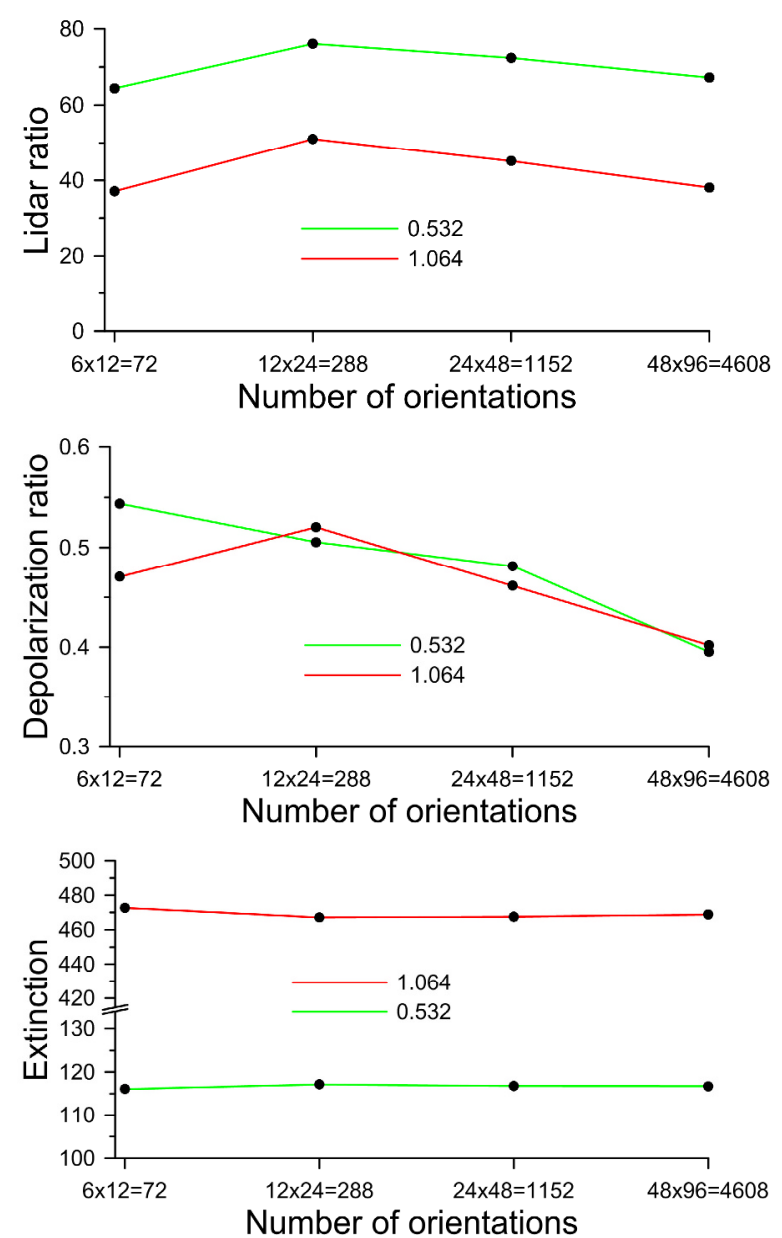

Figure 3. The lidar and depolarization ratio, and extinction coefficient $v$ s. number orientations.

\section{CONCLUSION}

Thus, it is shown that the physical optics approximation can be efficiently used to solve the problem of light scattering of large dust particles with arbitrary shape. The only condition is that the particle has to be faceted. This solution does not take the absorption effect into account for simplicity. The physical optics approximation significantly exceeds the exact numerical methods in speed. The results presented in the report are obtained on a desktop computer. It is also shown that the interference oscillations are not significant in case of dust aerosol particles; and the solution in the exact backscattering direction obeys power laws, as was shown for atmospheric ice crystals, which significantly simplifies the solution of the problem.

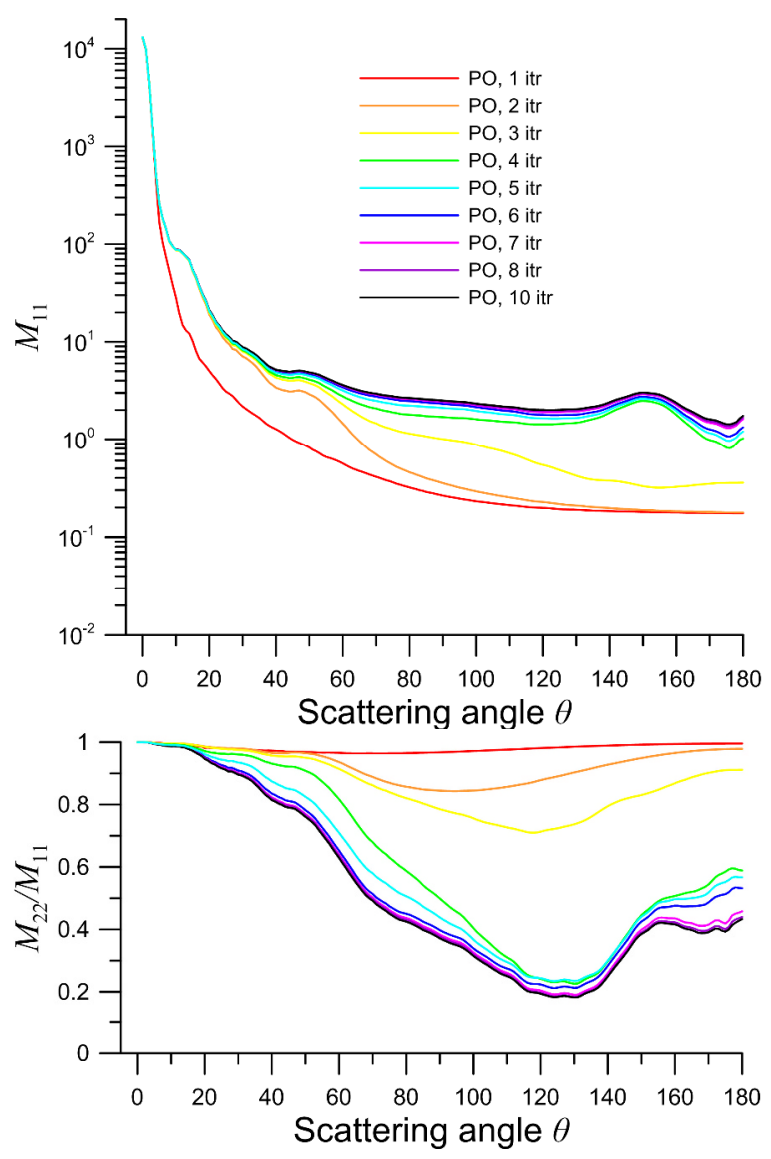

Figure 4. Element of the Mueller matrix depending on the number of iteration.

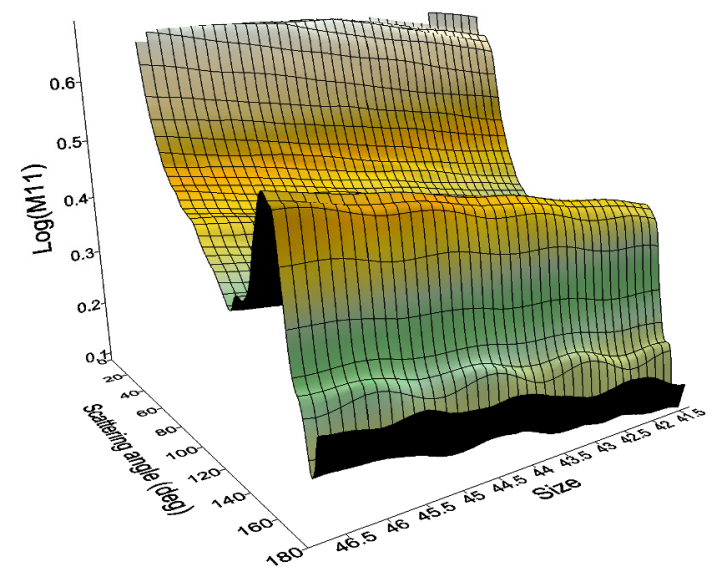

Figure 5. The interference oscillation of the first element of the Mueller matrix in the backscattering direction. 


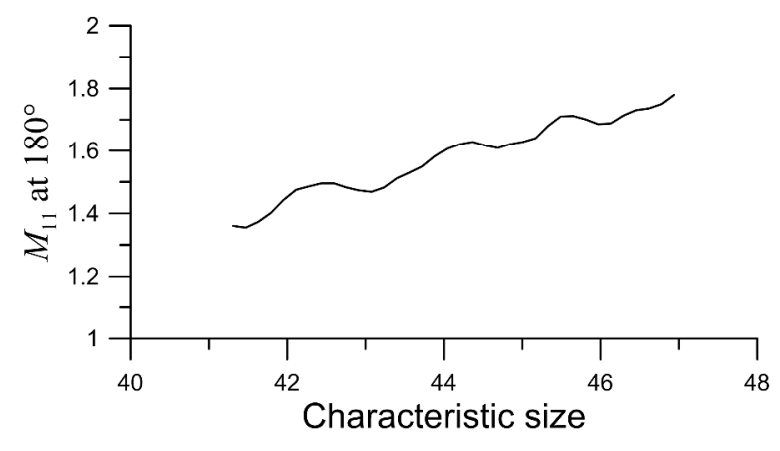

Figure 6. The interference oscillation
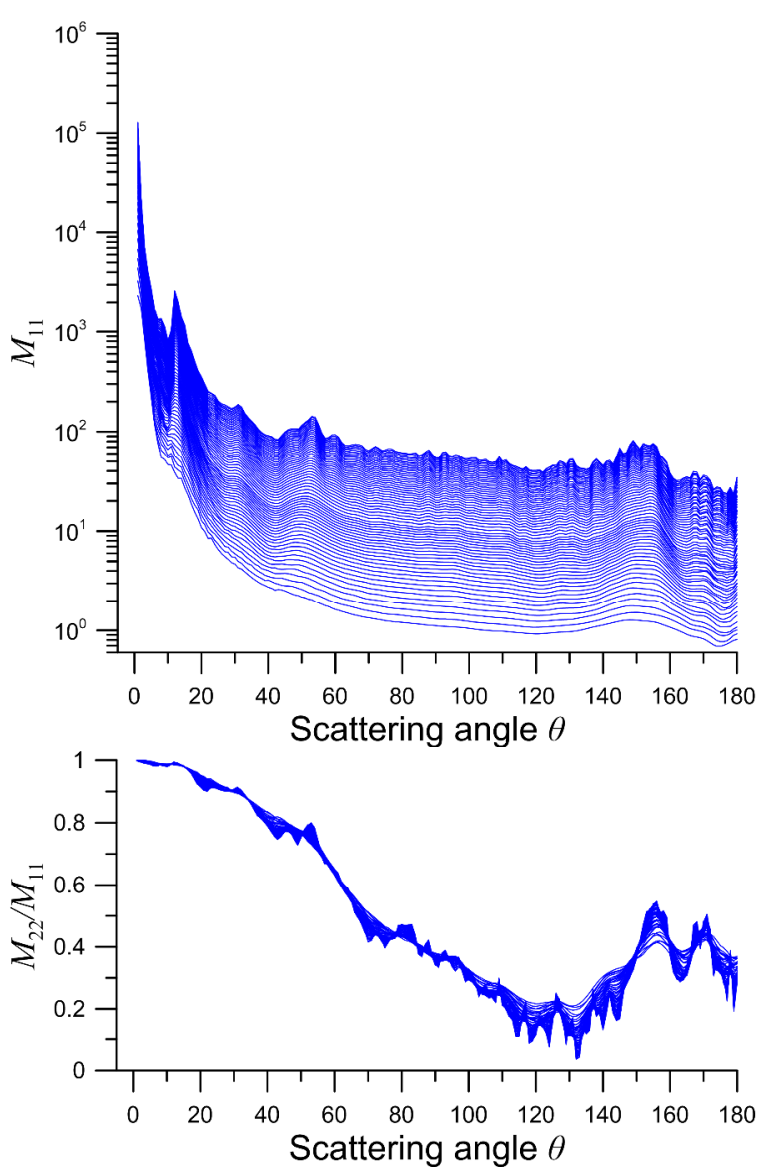

Figure 7. The elements of the Mueller matrix for a dust particle with size from 40 to 220.

\section{ACKNOWLEDGEMENTS}

This work is supported by the RFBR under Grants no. 18-05-00568, no. 18-55-53046, and budget project AAAA-A17-117021310145-6. Authors acknowledge PRACE for awarding access to MareNostrum at Barcelona Supercomputing Center (BSC), Spain, and GRNET for awarding computational time in the National HPC facility -
ARIS - under project ID pa170906-ADDAPAS and pr005038-REMOD. Calculation of Mueller matrixes within the PO is supported only by Russian Science Foundation (18-77-10035).
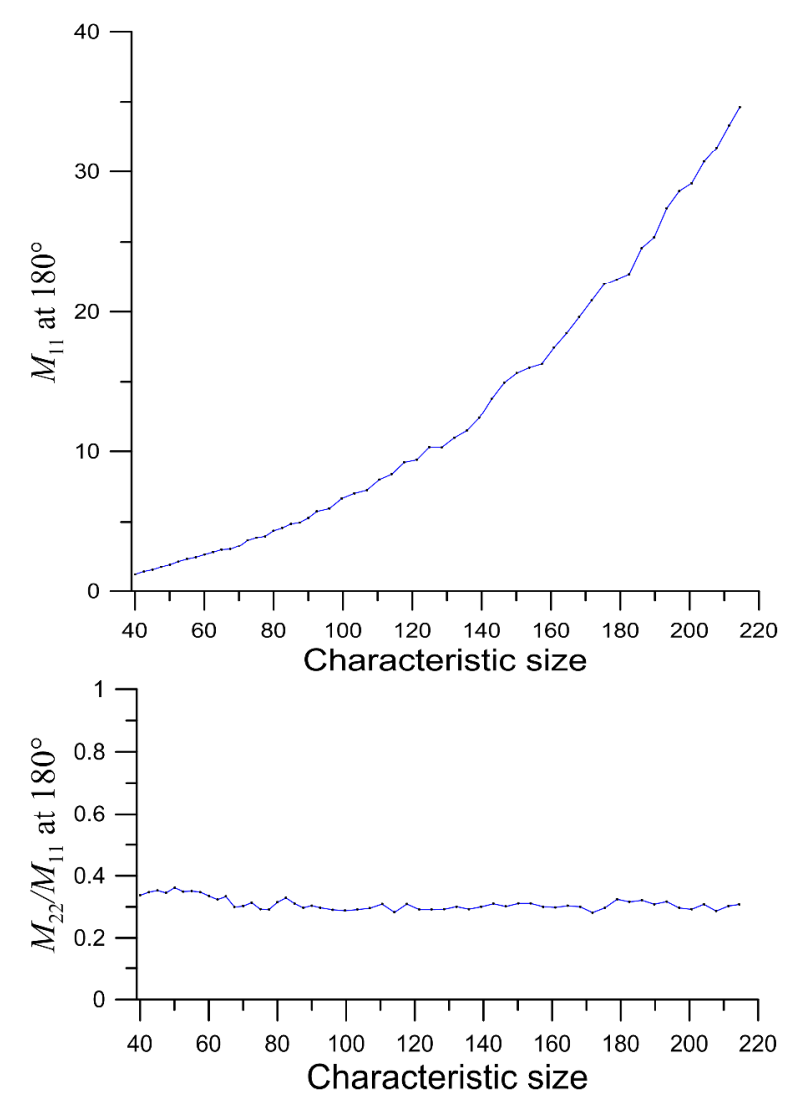

Figure 8. The elements of the Mueller matrix at exact backscattering direction.

\section{REFERENCES}

[1]J. Gasteiger, M. Wiegner, S. Groß, V. Freudenthaler, C. Toledano, M. Tesche, K. Kandler, Tellus B: Chemical and Physical Meteorology 63(4), 725-741 (2011)

[2] M. A. Yurkin， V. P. Maltsev， A. G. Hoekstra， J. Quant. Spectrosc. Radiat. Transfer 106, 546-557 (2007)

[3]A. Borovoi, A. Konoshonkin, N. Kustova, J. Quant. Spectrosc. Radiat. Transfer 146, 181-189 (2014)

[4]A. V. Konoshonkin, A. G. Borovoi, N. V. Kustova, H. Okamoto, H. Ishimoto, Y. Grynko, J. Förstner, J. Quant. Spectrosc. Radiat. Transfer 195, 132-140 (2017)

[5]A. V. Konoshonkin, N. V. Kustova, A. G. Borovoi, J. Quant. Spectrosc. Radiat. Transfer 164, 175-183 (2015)

[6] A. V. Konoshonkin, A. G. Borovoi, N. V. Kustova, J. Reichardt, Optics Express 25, 22341-22346 (2017) 